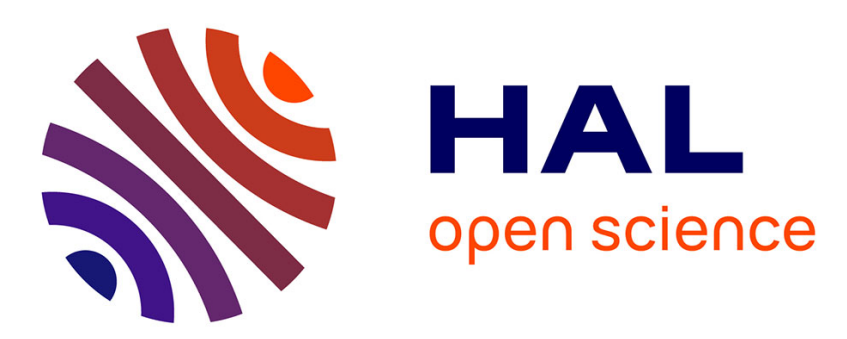

\title{
L'auteur scientifique en question: pratiques en psychologie et en sciences biomédicales
}

\author{
David Pontille
}

\section{To cite this version:}

David Pontille. L'auteur scientifique en question: pratiques en psychologie et en sciences biomédicales. Social Science Information, 2001, 40 (3), pp.433-453. 10.1177/053901801040003004 . halshs$00261741 \mathrm{v} 2$

\section{HAL Id: halshs-00261741 \\ https://shs.hal.science/halshs-00261741v2}

Submitted on 19 Apr 2020

HAL is a multi-disciplinary open access archive for the deposit and dissemination of scientific research documents, whether they are published or not. The documents may come from teaching and research institutions in France or abroad, or from public or private research centers.
L'archive ouverte pluridisciplinaire HAL, est destinée au dépôt et à la diffusion de documents scientifiques de niveau recherche, publiés ou non, émanant des établissements d'enseignement et de recherche français ou étrangers, des laboratoires publics ou privés. 


\section{L'auteur scientifique en question : pratiques en psychologie et en sciences biomédicales}

David Pontille*

chargé de recherche CNRS

pontille@univ-tlse2.fr 


\section{Résumé}

L'inflation des articles cosignés pose avec force la question de l'évaluation individuelle des chercheurs. Celle-ci est renforcée par l'augmentation des cas de fraude qui en retour soulève le problème de la responsabilité dans la production scientifique. La notion d'auteur est précisément façonnée par ces registres du crédit et de la responsabilité. Mais, parce que l'appropriation en science s'opère sous la forme spécifique de la paternité, elle cadre mal avec la définition juridique de l'auteur et de la propriété intellectuelle. Pourtant l'activité scientifique est traversée par des formes d'appropriation qui impliquent les registres de l'auctorialité (crédit et responsabilité). Partant d'une analyse des définitions pratiques de l'auctorialité dans deux espaces disciplinaires (la psychologie et les sciences biomédicales), ce texte propose des lignes de problématisation de l'auteur en science.

\section{Mots clés}

Auteur - Paternité scientifique - Responsabilité - Crédit - Signature 


\section{La question de l'auteur en science : une aporie}

Le nombre et le lieu de publication sont des éléments importants dans la carrière académique d'un chercheur. Lorsque celui-ci est l'unique auteur d'une publication, son implication dans la recherche est relativement peu ambiguë. Mais l'inflation du nombre d'auteurs par article dans une majorité d'espaces disciplinaires ${ }^{1}$ soulève de manière récurrente la question de l'évaluation individuelle des chercheurs (Stephan et Levin, 1997). Comment discerner la part relative de chacun des coauteurs ? Comment estimer qui a fait quoi ? Les pratiques individuelles étant spécifiques à chaque situation, elles sont multiples et complexes (Zuckerman, 1968 ; Pontille, 2000). Il arrive que certains prétendants à la signature soient ignorés tandis que d'autres sont « invités ", ajoutés alors qu'ils n'ont rien fait (honorary, ghost ou guest authorship). La prise en charge d'une même opération de recherche ${ }^{2}$ ne garantit pas, d'une publication à l'autre, que le nom d'un chercheur s'inscrive dans le même espace graphique (la liste des auteurs ou les remerciements). La question désignative « qui est l'auteur ? » suppose donc un accord sur les opérations qui sont jugées plus importantes, sur celles qui permettent d'être auteur, et sur les combinaisons d'opérations qui sont jugées suffisantes pour basculer des remerciements (subauthorship) à la liste des auteurs (authorship). Mais au-delà de ce travail de mise en ordre, la désignation présuppose également un accord sur la définition : qu'est-ce qu'un auteur?

Les qualités de la notion sont complexes : elles sont intimement liées à l'apposition autographe du nom propre (Fraenkel, 1992) et au développement de l'imprimé (Eisenstein, 1991 ; Chartier, 1996). Plusieurs registres construits historiquement définissent la notion. La conception commune est celle qui s'est mise en place au XVIIIe siècle dans le domaine juridique. Elle est concomitante à l'élaboration de lois qui confèrent des droits d'auteur, et réfère à la création originale strictement individuelle. Être auteur, c'est être créateur de formes et avoir des droits sur ces formes. Cette définition juridique de l'auteur insiste sur le lien marchand qui l'unit à ses productions. Celui-ci suppose une limite à l'appropriation par un tiers en garantissant un droit de propriété privée et donc de copie. Mais la notion d'auteur est simultanément chargée d'une autre signification, beaucoup plus ancienne : la conception diplomatique. Celle-ci «émane des chancelleries et s'appuie sur une conception de l'écrit comme moyen d'agir » (Fraenkel, 1997: 265). Être auteur, c'est dans ce cas avoir la capacité de décision et d'action. La notion renvoie ici à l'autorité conférée par l'écrit, elle engage la responsabilité et oblige à assumer les conséquences de ses actes écrits ${ }^{3}$.

\footnotetext{
1. Tout au long de ce texte, la notion d'« espace disciplinaire » est utilisée pour mettre l'accent sur un double processus qui caractérisent les « disciplines " et les "spécialités " : celui du cadrage des enjeux, et celui des jeux aux frontières. Elle désigne donc la capacité d'encadrements épistémiques et pratiques des activités de recherche tout en accordant une place au frottement entre spécialités ou disciplines. Sur cette notion, voir Pontille (2000, chap. 4).

2. Plusieurs tâches sont généralement distinguées : être à l'origine de l'idée de la recherche et/ou de la publication ; rechercher un financement, être responsable du laboratoire ; organiser et superviser le déroulement du projet et/ou du manuscrit ; planifier et concevoir les méthodes d'investigation empirique ; réaliser les expériences, la collecte des matériaux empiriques ; mettre en ordre les résultats et produire du sens ; faire l'état de la question ; écrire la totalité ou une partie importante de la publication ; retravailler l'écriture du manuscrit sur le fond et sur la forme.

3 . Le pseudonymat ou l'anonymat révèlent la force de cet engagement. Certains livres ne sont pas signés du nom de leur auteur pour échapper à la censure ou au discrédit engendrés par des écrits qui seraient indignes de son rang ou de sa profession. Sur ce point voir Foucault (1969) ; Couturier (1995).
} 
Si dans ces deux conceptions la notion d'auteur désigne une forme de présence dans les objets produits, la première met l'accent sur le crédit qu'en retire le créateur tandis que la seconde privilégie la responsabilité du contenu de l'écrit. Cependant crédit et responsabilité sont considérés comme absolus dans les deux cas. Le premier n'est pas transférable et la seconde n'est pas divisible. La présence dans les objets doit donc émaner d'une figure également absolue et indivisible : une personne réelle singulière, et non une persona ficta. Acte physique et intentionnel, la signature garde les traces de cette présence et assure le lien avec la personne (Fraenkel, 1992).

À ce niveau, l'activité scientifique présente des spécificités. D'une part, le crédit s'y manifeste d'une manière particulière : le contenu du travail (la nature ou le social) n'est par définition pas soumis au régime de la propriété privée ${ }^{4}$. Les chercheurs transfèrent leurs droits à la communauté scientifique et leurs productions appartiennent au domaine public (Merton, 1968 ; Zuckerman et Merton, 1971 ; Hagstrom, 1982). La forme (au sens juridique) pourrait faire l'objet d'une appropriation personnelle - comme en peinture ou en littérature. Mais en science, la forme appartient à la catégorie opposée à la vérité : revendiquer la forme reviendrait à s'approprier un artefact. Opérant par le seul attachement du nom du chercheur à ses productions, le mode de valorisation est strictement symbolique. D'autre part, la responsabilité n'est pas conçue comme totale : les pratiques de production s'accomplissent sur le mode collectif. Elles conduisent de la sorte à une distribution différentielle de celle-ci dans chaque projet de recherche. Cette collectivisation est d'autant plus forte qu'elle s'organise dans de nombreux cas au sein de groupes de recherche internationaux (Luukkonen et al., 1992). À ce niveau, la responsabilité est répartie entre plusieurs personnes dispersées en divers lieux. Ainsi, la dimension absolue de la responsabilité ne peut avoir cours, et la notion d'auteur est mise en défaut. Tant les procédures de création originale que celles de certification ne résultent donc pas d'une personne individuelle unique.

Du fait de ces particularités, l'appropriation en science s'opère sous le régime spécifique de la paternité (authorship), et « le scientifique est littéralement un non-auteur » (Biagioli, 1999: 14). Pourtant la publication scientifique donne lieu à des procédures d'appropriation qui impliquent les registres de l'auctorialité : en signant leurs productions, les chercheurs veulent garantir les bonnes attributions (le crédit) et certifier les connaissances par la publication (la responsabilité). Mais en pratique les notions de crédit et de responsabilité peuvent être définies et articulées de diverses manières autour des conceptions de l'auteur esquissées précédemment. Deux lignes de tension se dégagent alors : celle qui met en jeu le pôle du crédit et le pôle de la responsabilité, et celle qui oppose la notion d'auteur à celle - à construire - d'« auteur collectif », et plus précisément ici d'« auteur scientifique ».

Ce texte questionne les spécificités de l'auteur en science, et étudie la manière dont ces tensions s'expriment dans les pratiques de deux espaces disciplinaires. L'analyse s'appuie sur un premier dépouillement de données qui concernent différents documents de psychologie et de sciences biomédicales : des codes d'éthique, des manuels de style, ainsi que des éditoriaux, des lettres, des articles, et des instructions aux auteurs publiés dans plusieurs revues (American Psychologist, Annals of Internal Medicine, British Medical Journal, New England Journal of Medicine, Journal of the American Medical Association, the Lancet). Bien qu'elle se fonde sur une analyse de contenu de l'ensemble de ces

\footnotetext{
4. Laissons de côté momentanément le dépôt de brevets qui s'accroît considérablement depuis une vingtaine d'années dans le domaine des biotechnologies.
} 
documents (59 textes, soit environ 750 pages), l'étude portera essentiellement sur les dernières versions du Publication Manual édité en 1983 et 1994 par l'American

Psychological Association (APA), ainsi que sur les prescriptions éditoriales émises par I'International Committee of Medical Journal Editors (ICMJE) entre 1985 et 2000. L'accent sera mis sur la manière dont sont définis et articulés les registres de l'auctorialité dans ces guides de publication afin de proposer, en conclusion, des lignes de problématisation de l'auteur en science.

\section{La psychologie : les qualités de l'auteur garanties par la signature}

La psychologie incarne d'une certaine façon l'adoption réussie de la méthode expérimentale au sein des sciences sociales. En quelque sorte, elle constituerait le modèle à suivre par ces sciences, qui, du fait de sa légitimité hégémonique après la Seconde Guerre mondiale aux Etats-Unis, se voient d'ailleurs renommées behavioral sciences :

La psychologie expérimentale a été la première science humaine à établir un discours spécialisé, qui se distingue du discours traditionnel de la philosophie. La psychologie expérimentale est devenue le modèle et a fixé les standards pour toutes les spécialités de la psychologie qui aspiraient au statut de science. (Bazerman 1988: 259)

Cette influence s'est construite historiquement par l'intermédiaire des différentes versions 5 d'un code d'éthique (APA, 1992) et du Publication Manual (APA, 1994).

\section{Le Publication Manual comme cadre performatif}

Les premières prescriptions émergent au moment où le programme behavioriste commence à dominer la psychologie expérimentale. Elles datent de février 1929, et sont publiées dans un article de six pages et demie. La standardisation qu'elles opèrent prend en compte l'ensemble des éléments qui constituent une publication scientifique écrite ou orale. De la formulation des phrases, jusqu'à la ponctuation, la métrique de calcul, les abréviations pour la correction par les referees, en passant par les titres et sous-titres, les tableaux, les graphiques, le résumé, les références ou les remerciements : rien n'est laissé au hasard. La dernière édition en date (1994) reprend l'ensemble des prescriptions de la version précédente (1983) en ajoutant de nombreux exemples et annexes qui portent cette quatrième édition à trois cent soixante-huit pages.

L'American Psychological Association exerce donc une activité normative tant au niveau du contenu qu'à celui du format d'écriture des articles de psychologie. Le Publication Manual qu'elle édite se donne à voir sous le registre de l'énoncé performatif : en normalisant les règles d'écriture relativement présentes dans les pratiques, il modifie, transforme et radicalise tant les pratiques de publication des résultats que les pratiques de recherche (le primat étant accordé aux études empiriques). La normalisation de l'écriture s'accompagne d'une normalisation des procédures expérimentales, et vice-versa.

La paternité des publications est l'objet d'une section spécifique : characteristics of authorship. Les prescriptions qu'elle contient concernent les opérations de recherche qui donnent accès à la liste des auteurs. Ces opérations de recherche sont hiérarchisées selon

\footnotetext{
5. Le code d'éthique a fait l'objet de neuf versions à partir de sa première élaboration en 1953, et le Publication Manual en compte huit depuis février1929.
} 
les principes éthiques des psychologues érigés en code de conduite (Spiegel et KeithSpiegel, 1970 ; Bridgwater et al., 1981).

La paternité est réservée aux personnes qui reçoivent le crédit principal et tiennent la responsabilité essentielle pour le travail publié. La paternité inclut, par conséquent, non seulement ceux qui participent à l'écriture mais aussi ceux qui ont fait des contributions scientifiques substantielles à l'étude. (APA, 1994: 294)

Dans cet espace disciplinaire, où la pratique expérimentale est centrale, l'écriture est certes une opération de recherche importante, mais elle n'est pas primordiale. Plusieurs autres contributions sont considérées substantielles. Elles peuvent inclurent

le problème ou les hypothèses, la conception du montage expérimental, l'organisation et la conduite de l'analyse statistique, l'interprétation des résultats, ou l'écriture d'une majeure partie de l'article. Ceux qui contribuent de la sorte sont dans la liste. Les contributions moins importantes, qui ne relèvent pas de la paternité, peuvent être remerciées en note. Ces contributions peuvent inclure des fonctions de soutien comme la conception ou la construction des appareils, la collection ou la saisie des données, la modification ou la construction d'un programme informatique, et le recrutement de participants ou l'obtention d'animaux. (APA, 1994: 294)

La responsabilité prise en charge dans le projet est donc rendue visible par l'espace d'inscription du nom. Les opérations qui concernent une contribution plutôt intellectuelle sont valorisées, alors que celles qui concernent un apport plutôt manuel assignent aux remerciements ${ }^{6}$. Mais la quantité de travail intervient également : la responsabilité de plusieurs tâches qui assignent aux remerciements « peut cependant justifier le partage de la paternité » (APA, 1994: 295). Ainsi, il est recommandé aux divers participants d'une recherche de décider dès que possible " des tâches nécessaires pour la réalisation du projet, de la division du travail, des tâches ou des combinaisons de tâches qui méritent la paternité et du niveau auquel le crédit est accordé (premier auteur, deuxième auteur, etc) » (APA, 1994: 295). Les opérations de recherche étant hiérarchisées selon un code de conduite, l'ordre des signatures est également codifié.

La règle générale est que le nom du principal contributeur devrait apparaitre en premier, avec les autres noms dans l'ordre décroissant des contributions. Si des auteurs jouent des rôles équivalents dans la recherche et la publication de leur étude, ils peuvent le spécifier dans le second paragraphe de la première note. (APA, 1994: 295)

Cet agencement des noms s'opère donc selon la responsabilité intellectuelle impliquée dans le projet de recherche, et décroît à partir de la première place. De ce fait, l'ordre des signatures reflète la responsabilité et l'implication dans la recherche quel que soit le statut hiérarchique des auteurs (Over et Smallman, 1973 ; Fine et Kurdek, 1993) :

La paternité principale et les autres crédits de la publication reflètent avec précision les contributions scientifiques ou professionnelles relatives des individus impliqués, sans tenir compte de leur propre statut. La simple possession d'une position institutionnelle, comme celle de responsable de département (ou de directeur de laboratoire) ne justifie pas le partage

6. Cette distinction entre contribution cognitive et tâche manuelle n'est pas nouvelle. Elle s'inscrit dans une tradition historique qui remonte aux fondements de la pratique expérimentale. Sur ce point voir Shapin $(1989,1994)$. 
de la paternité. Les contributions mineures à la recherche ou à l'écriture de la publication sont remerciées de manière appropriée, dans les notes de bas de page ou dans un compte-rendu introductif. (APA, 1992: principe 6.23b)

Cette mise en ordre des noms évacue du même coup la possibilité alphabétique, qui est très faiblement utilisée dans cet espace disciplinaire. Endersby (1996) a en effet montré sur un échantillon de 2321 articles que ce type de mise en ordre est très peu présent: seulement $18 \%$ de ceux cosignés par trois auteurs, $9 \%$ de ceux cosignés par quatre auteurs, et $2 \%$ de ceux cosignés par cinq auteurs.

Ces prescriptions cadrent donc les modalités d'accès à la liste des auteurs et d'agencement des noms sur la publication. Si dans l'ensemble elles sont similaires entre les deux dernières versions du Publication Manual (1983,1994), la question de l'auteur a par contre subi des transformations importantes.

\section{Contresigner la paternité : vers un dédoublement de la signature}

Ces modifications se caractérisent par un renforcement des mesures concernant la signature à trois niveaux. Tout d'abord, les instructions deviennent plus nombreuses d'une version à l'autre, et le ton se fait de plus en plus précis : si seulement une page est consacrée à la paternité dans la version de 1983 (section 1.03 p. 20), celle de 1994 aborde par contre cette question en plusieurs sections (1.03 p. $4 ; 1.15$ p. $21 ; 5.01$ p. $274 ; 6.05$ p. 292-295) représentant à peu près six pages au total. Ensuite, comme le stipule explicitement l'introduction de la section 6.05 , les principes sont érigés " pour atteindre deux objectifs : assurer l'exactitude de la connaissance scientifique, et protéger les droits de propriété intellectuelle » (APA, 1994: 294), mais également pour « éviter les incompréhensions et préserver la réputation professionnelle et les relations » (APA, 1994: 4). Enfin, la lettre qui présente l'article lors de sa soumission à une revue est dorénavant accompagnée :

Bien que ce ne soit pas demandé avant l'acceptation du manuscrit, l'APA exige dorénavant une certification écrite de la paternité. (APA, 1994: 256)

Cet acte de certification de la paternité consiste à authentifier l'ordre des noms. II passe donc par une double signature. La première est celle de l'article sur lequel figure le nom des auteurs. Imprimée, cette signature signifie un engagement physique et moral qui lie le document à ses auteurs par l'apposition de leur nom propre. Mais la certification requise par l'APA double cet acte d'authentification : c'est à ce niveau qu'intervient la seconde signature. Apposée par la main de son scripteur sur la lettre qui accompagne l'article, celleci est investie de tous les attributs du signe (Fraenkel, 1992) : la responsabilité et l'autoréférentialité sont sans équivoque. Dans cette lettre qui explique brièvement le contenu de l'article, les auteurs s'engagent à ne pas soumettre simultanément leur publication à une autre revue. Mais cette signature qui s'opère en coulisses engage ici à deux niveaux supplémentaires :

En signant cette partie du formulaire, chaque auteur (a) accepte la responsabilité des contenus de l'article publié et (b) approuve l'ordre des signatures. Chaque auteur qui est nommé dans la liste doit signer la certification de la paternité. Les signatures photocopiées ou faxées sont acceptables pour tous, excepté le corresponding auteur, dont la signature doit être originale. (APA, 1994: 274) 
Chacun des coauteurs est donc tenu de signer pour valider son adhésion au contenu de l'article, mais également à l'ordre des signatures sur l'article. Si l'imprimé sépare la main du scripteur de son écrit par la typographie standardisée, l'engagement certifié est alors maintenu par la signature manuscrite avant la publication imprimée de l'article. Et pour authentifier cette responsabilité, la signature ne doit faire l'objet d'aucun support intermédiaire (photocopie ou fax) : elle doit être " originale ", au moins pour le responsable du projet (le corresponding auteur). Par ce double engagement des auteurs, l'APA entend éviter tout conflit en matière de paternité : les auteurs sont responsables du contenu des articles, et ils acceptent l'ordre des signatures qui y figure.

La négociation de l'ordre des noms s'inscrit donc dans une double articulation. La première concerne les grandes lignes que prescrivent le Publication Manual et le code de conduite, déterminant les opérations de recherche qui assignent soit à l'espace des auteurs, soit à celui des remerciements. La seconde se déploie à l'échelle de chaque situation particulière où s'agencent avec plus ou moins de facilité les prescriptions générales. De ce fait, bien qu'elle soit définie à un niveau typique, la division hiérarchisée de la responsabilité est entre les mains des divers collaborateurs, et doit être acceptée par tous.

Les auteurs sont responsables de la détermination de la paternité et de la spécification de l'ordre des noms de deux auteurs et plus dans la liste. (...) Le corresponding auteur (...) devrait toujours obtenir le consentement d'une personne avant d'inclure son nom dans la liste ou dans la note. Chaque auteur figurant dans la liste d'un article devrait relire (review) la totalité du manuscrit avant qu'il soit soumis. (APA, 1994: 295)

En psychologie, s'engageant sur le contenu de l'article et sur l'ordre des noms par leur double signature, les auteurs sont tenus responsables de cet agencement au cas par cas. En faisant lire le contenu de la publication et en faisant signer une certification de la paternité par chacun des coauteurs, l'APA vise à minimiser la pratique des auteurs invités. Tant le registre du crédit que celui de la responsabilité sont clairement définis et articulés. Le premier se régule par la mise en ordre décroissante des contributions substantielles dans les signatures, et le second est mis en œuvre par la signature manuscrite du certificat.

La construction de ces repères pour la prétention à la paternité et la mise en ordre des noms est donc une tentative pour cadrer les pratiques individuelles et hétérogènes.

Cependant, " de telles lignes directrices peuvent être interprétées de façons différentes et souvent conflictuelles, selon la profession, les expériences, les valeurs, le statut et le pouvoir de la personne qui interprète, et sur ce qu'elle pourrait gagner ou perdre en appliquant des interprétations particulières " (Digiusto, 1994: 55). C'est en partie pourquoi d'autres espaces disciplinaires, comme les sciences biomédicales, proposent d'aborder les procédures de mise en ordre d'une toute autre façon.

\section{Les sciences biomédicales : vers la fin de l'auteur ?}

L'inflation importante du nombre d'auteurs par article en sciences biomédicales a conduit relativement tôt à l'élaboration de repères pour agencer la liste des signataires. Le problème principal qui s'est posé concerne la responsabilité des coauteurs en cas d'identification d'une fraude (plagiat, trucage ou invention des données). 


\section{Réhabiliter la responsabilité face à la fraude}

Plusieurs affaires frauduleuses - notamment celle de John Darsee (Huth, 1986 ; Broad et Wade, 1987), de Robert Slutsky (Engler et al., 1987), et plus récemment le cas "Baltimore » - ont en effet révélé que les coauteurs ne se sentaient pas responsables, rétorquant que l'inclusion de leur nom était une formalité ou une politesse alors qu'ils n'avaient pas effectivement participé à la recherche. L'importance de la pratique des auteurs invités dans les sciences médicales (Rennie et Flanagin, 1994 ; Smith, 1994) peut donc conduire à une impasse : les auteurs, qui s'engagent en signant, déclinent ensuite toute responsabilité en cas de fraude. Ils sont prêts à cosigner pour le meilleur (le crédit), mais surtout pas pour le pire (le discrédit). Or, en facilitant la publication d'articles frauduleux, la pratique “ d'invitation » a d'autres conséquences. D'une part, elle discrédite à la fois ceux qui ont réellement participé à la recherche et les éditeurs des revues qui ont publié ces articles. D'autre part, les coauteurs invités sont incapables de répondre du contenu de la publication qu'ils ont signé, lorsqu'un collègue leur demande des précisions techniques ou théoriques sur les expériences.

C'est pour contrer ces dérives qu'en 1985, I'International Committee of Medical Journal Editors a constitué un ensemble de critères qui donne accès à la paternité (ICMJE, 1985). Cette politique éditoriale prescrivait notamment de réduire le nombre d'auteurs à six maximum, en listant, y compris dans les références de la bibliographie, les six auteurs suivi de « et al. » lorsque l'article en comprenait sept et plus. De cette façon, le « senior investigator ", souvent placé en dernière position, disparaissait à chaque fois que les cosignataires étaient supérieurs à six. La méthode choisie était donc indirecte. Elle en appelait à la responsabilité des personnes réellement impliquées dans le travail faisant l'objet de la publication. Dans la version de 1993 des exigences de l'ICMJE, les critères sont les suivants :

- Toutes les personnes désignées comme auteur devraient remplir les conditions pour la paternité. L'ordre des signatures devrait être une décision commune des coauteurs. Chaque auteur devrait avoir suffisamment participé au travail pour répondre d'une responsabilité publique du contenu.

- La paternité devrait être fondée uniquement a) sur des contributions substantielles à la conception et l'élaboration, ou l'analyse et l'interprétation des données ; et b) sur l'écriture du premier jet de l'article ou sa révision critique au niveau du contenu intellectuel ; et c) sur l'approbation sur la version finale en voie de publication. Les conditions a), b), c) doivent toutes être remplies. La seule participation à l'acquisition des fonds financiers ou à la collection des données ne justifie pas la paternité. La supervision générale du groupe de recherche n'est pas suffisante pour la paternité. Toute partie d'un article, cruciale pour ses conclusions majeures, doit être la responsabilité d'au moins un auteur.

- Les responsables de revues peuvent exiger des auteurs qu'ils justifient l'attribution de la paternité. (ICMJE, 1993)

Du point de vue de la responsabilité, ces critères sont très proches de ceux qui sont définis par l'APA. Ils ont été acceptés et adoptés par environ cinq cents revues de sciences biomédicales. Et parce qu'elles figurent dans les instructions aux auteurs de ces revues, les prescriptions de l'ICMJE sont certainement « la définition de la paternité la plus largement diffusée dans le champ biomédical » (Leash, 1997). Érigés au rang de prescriptions, les repères pour la prétention à la paternité deviennent incontournables : ils sont des normes auxquelles doivent se plier les auteurs. Epstein (1993) a d'ailleurs montré que les pratiques 
de signature s'y conforment plus ou moins sur la période 1982-1992 dans huit revues de sciences biomédicales (Cell, Nature, Proceedings of the National Academy of Sciences, Journal of Clinical Investigation, Biochemical and Biophysical Research Communications, Journal of Clinical Oncology, New England Journal of Medicine, Lancet).

Cependant, d'autres études plus récentes ont montré que les pratiques de signature de cet espace disciplinaire diffèrent des normes de l'ICMJE (Goodman, 1994 ; Shapiro et al., 1994 ; Eastwood et al., 1996 ; Bhopal et al., 1997 ; Drenth, 1998 ; Flanagin et al., 1998 ; Hoen et al., 1998 ; Yank et Rennie, 1999). En Angleterre, Goodman (1994) a envoyé un questionnaire à l'ensemble des coauteurs de 12 articles en combinant des critères qui sont prescrits par l'ICMJE (sans les identifier) et plusieurs autres. Parmi les 84 auteurs interrogés sur leurs contributions et celles de leurs coauteurs, un tiers déclare ne pas avoir fait de contribution substantielle au contenu intellectuel des articles. Dans une étude plus vaste aux Etats-Unis, concernant 200 auteurs ayant publié en premier nom dans 10 revues scientifiques majeures, Shapiro et al. (1994) ont également montré que $26 \%$ de la totalité des auteurs n'ont pas contribué substantiellement aux tâches intellectuelles de concept, conception, analyse, interprétation, écriture et/ou relecture critique. Eastwood et al. (1996) rapportent des résultats similaires à partir d'un questionnaire adressé en 1992 à 324 chercheurs post-doctorants de l'université de Californie à San-Francisco. Une majorité d'entre eux considéraient, en accord avec les exigences de l'ICMJE, que certaines opérations de recherche conduisent inévitablement à la signature de l'article : analyser et interpréter les données (92\%), écrire le premier jet de l'article (69\%), développer ou collaborer à l'élaboration d'une hypothèse (65\%). Toutefois, d'autres opérations, exclues des critères pour la paternité par l'ICMJE, étaient fortement représentées : construire les expériences ou collecter les données (85\%), être le directeur du laboratoire dans lequel le travail a été effectué (47\%), obtenir les fonds pour le projet de recherche (44\%). De plus, $37 \%$ des post-doctorants déclaraient avoir été sollicités pour ajouter quelqu'un qui n'a rien fait sur un article, si cela pouvait favoriser la publication de la recherche ou être bénéfique pour leur carrière. Plus récemment Bhopal et al. (1997) ont montré que peu de chercheurs connaissaient les critères de l'ICMJE, et que dans le cas contraire, ils ne les approuvaient pas $(62 \%)$ et reconnaissaient la pratique des auteurs invités utile pour maintenir de bonnes relations de travail.

La conformité aux normes prescrites à un niveau professionnel international est donc contrecarrée dans les pratiques à deux niveaux au moins. D'une part, de nombreux chercheurs interrogés déclarent ne pas les appliquer strictement ou ne pas les comprendre.

Les critères de l'ICMJE ont été développés sur les bases de débats de principes, en réponse à la croissance des auteurs invités et de la fraude en science. lls ont été largement publiés, mais dans notre étude de nombreux chercheurs les ignorent, tendent à mal les comprendre, et les perçoivent comme ouverts à de mauvaises interprétations. (Bhopal et al., 1997)

D'autre part, les perceptions et les conceptions sont diverses dans cet espace disciplinaire aux frontières de la biologie, de la chimie et de la médecine :

Par exemple, certains admettent que le dernier auteur d'un article est celui qui a le moins contribué, tandis que d'autres considèrent que le dernier auteur est un chercheur confirmé qui a le plus contribué à l'élaboration conceptuelle. (Digiusto, 1994: 55)

Espace de recherches interdisciplinaires, les sciences biomédicales donnent lieu à des pratiques de mise en ordre des signatures qui sont héritées de traditions disciplinaires 
différentes, et les opérations considérées comme décisives pour la paternité varient " d'un département à l'autre, d'une institution à l'autre, d'une spécialité à l'autre » (Leash, 1997), malgré l'élaboration d'un cadre normatif international commun.

\section{Contribution contre paternité : la dissolution de l'auteur}

Face à cette diversité et à l'absence d'application des critères de l'ICMJE, une conférence, regroupant chercheurs, responsables de revues, et administrateurs de recherche, s'est tenue à Nottingham en juin 1996 pour redéfinir la paternité scientifique. Elle a été complétée par une autre conférence en février 1998 à Berkeley. La question principale de ces deux conférences est claire (Godlee, 1996 ; Horton et Smith, 1996 ; Smith, 1997b) : faut-il renouveler la paternité dans les publications scientifiques?

Une première solution consisterait à diffuser encore plus largement les critères de l'ICMJE. Mais n'étant pas reconnus et légitimés, leur application reste incertaine. Une autre proposition a alors émergé de ces journées de réflexion. Elle consiste à inclure dans chaque article une note de bas de page dans laquelle chaque auteur définit la contribution spécifique qui lui revient dans le projet de recherche - une solution proposée à maintes reprises (Moulopoulos et al., 1983 ; Saffran, 1989 ; Goodman, 1994 ; Shapiro et al., 1994). La responsabilité des coauteurs est ainsi rendue visible par l'identification des opérations de recherche qu'ils ont pris en charge. Tant les lecteurs que les responsables de revues peuvent donc identifier et attribuer la part relative du travail de chacun. Cette solution a notamment conduit à la modification du dernier critère de l'ICMJE :

- Les responsables de revues peuvent demander aux auteurs de décrire la contribution de chacun : cette information peut être publiée. (ICMJE, 1997)

La responsabilité n'est donc plus laissée dans l'ombre, comme en 1993 où les responsables de revues pouvaient demander aux auteurs de « justifier l'attribution de la paternité ». Ceuxci peuvent dorénavant les obliger à expliciter dans le texte les fondements de l'ordre des noms. Une solution d'ailleurs adoptée en 2000 :

- Les auteurs doivent fournir une description de la contribution de chacun, et les responsables de revues doivent publier cette information. (ICMJE, 2000)

Similaire à la certification de la paternité prescrite en psychologie, le renseignement d'un formulaire spécifiant la contribution de chacun s'opère dorénavant en coulisses, avant la publication. Les auteurs sont tenus d'y spécifier leurs contribution(s) respective(s) qui sont désormais codifiées ${ }^{7}$. En signant de leur main ce formulaire qui précise leur(s) niveau( $x$ ) d'implication dans la recherche, chacun des participants engage ainsi sa propre responsabilité concernant la partie du travail qu'il a endossé.

Cette pratique permet donc de lever le voile sur la participation effective de chacun des cosignataires d'une publication. La question récurrente du « qui a fait quoi » est ici abordée de front. La décomposition de la responsabilité, engendrée par la division du travail, ne se retrouve pas seulement ordonnée dans la cosignature des participants : elle est détaillée et

7. (a) concept et conception, (b) analyse et interprétation des données, (c) écrire la première version de l'article, (d) révision critique de l'article sur le contenu, (e) approbation finale de l'article, (f) apport des matériaux ou des patients d'étude, (g) Expertise statistique, (h) obtention de financements, (i) support administratif, technique, ou logistique, (j) recueil et mise en forme des données (« Information for Authors » Annals of Internal Medicine 2000). 
attribuée à chacun. La pratique des auteurs invités est donc relativement évacuée. Les modalités de mise en ordre des signatures scientifiques relèvent toujours du choix des coauteurs. Mais parce que leur contribution effective est publiée en note, une mise en ordre codifiée des noms n'a plus lieu d'être : quelle que soit sa place dans la liste, chaque coauteur signe et partage la paternité pour la prise en charge d'opérations de recherche dorénavant explicitées. Les collaborateurs doivent donc se mettre d'accord « sur la part relative de leurs contributions pour décider de l'ordre dans lequel [leurs noms] seront listés » (Smith, 1997a).

Si elle résout relativement le problème des auteurs invités, la description des opérations de recherche de chaque coauteur a néanmoins des conséquences importantes du point de vue de la notion d'auteur. Elle rend explicite la contribution de chaque participant qui, engagé et responsable d'une partie du processus de recherche, signe l'article. Chacun des « contributeurs » peut donc prétendre au partage de la paternité. Les coauteurs décident de l'ordre des noms (par décroissance des contributions ou selon un autre scénario), mais toute contribution qui apporte utilement au travail peut conduire à la signature de la recherche. La distinction entre travail cognitif et tâche non-cognitive se fait plus floue. La liste des auteurs devient de ce fait plus ouverte : ceux qui étaient à la marge, remerciés en notes, peuvent dorénavant y accéder plus facilement. Le qualificatif de " contributeur » découle donc de la solution qui consiste à décrire la contribution de chacun au projet final. D. Rennie et R. Smith, respectivement responsable du Journal of the American Medical Association et du British Medical Journal, s'en font d'ailleurs les fervents défenseurs en proposant de substituer cette notion (contributorship) à celle de paternité (authorship).

En sciences biomédicales, la paternité scientifique semble donc arrivée à un tournant, comme l'indiquent les titres des articles et éditoriaux de revues : " when authorship fails : a proposal to make contributors accountable » (Rennie et al., 1997), « authorship : time for a paradigm shift ? » (Smith, 1997b), « authorship is dying : long live contributorship » (Smith, 1997a). La notion de contributeur met en œuvre une décomposition de la responsabilité qui est alors distribuée. Elle signifie un engagement plus fort sur la contribution de chacun, mais plus sur l'ensemble de la recherche publiée. Si les registres du crédit et de la responsabilité sont rendus explicites pour chaque contributeur, la dimension totalisante de la responsabilité impliquée par la signature est par contre mise en défaut. Pour combler ce vide entre niveau individuel et prise de responsabilité du travail collectif, les directeurs de revues (Rennie et al., 1997 ; Smith, 1997a) proposent d'adjoindre à la notion de contributeur celle de « garant » (guarantor) : certains des contributeurs doivent assumer l'intégralité de l'article 8 .

\section{Les figures de l'auctorialité scientifique}

Afin de court-circuiter la pratique des auteurs invités et les possibilités de fraude qui se développent, les associations professionnelles et les comités d'éditeurs de revues élaborent des repères. Les diverses opérations de recherche sont alors hiérarchisées et divisées selon un cadre commun. C'est au cours de ce cadrage des pratiques de recherche et de

\footnotetext{
8. «Les contributeurs seront pleinement responsables de leur contribution, mais au moins une personne - le garant - doit accepter la responsabilité de l'ensemble du travail » (Smith, 1997a) ; "C'est souvent irréaliste de demander à l'ensemble des contributeurs de prendre la responsabilité de tous les aspects de l'étude. Certains des contributeurs doivent assurer l'intégrité de la totalité, et apparaître comme garants de l'article entier » (Rennie, 1999).
} 
signature que les registres de l'auctorialité sont définis et ajustés de manière spécifique. Dans ce travail de mise en ordre, un cadrage différentiel des pratiques est effectif.

En psychologie, l'auteur est conçu comme absolu : l'accent est mis davantage sur la responsabilité collective de la recherche. Chaque auteur est tenu responsable du contenu de l'ensemble de la publication et de l'ordre des noms à niveau égal. Le crédit est pour sa part réglé par l'agencement décroissant des contributions dans la liste des noms sur la publication. Dans cet espace disciplinaire, les prescriptions tendent donc à construire une définition collective de l'auteur qui opère à partir de la définition individualiste. Ainsi, la conception de l'auteur en psychologie garde en partie les traces de son ancrage dans les sciences humaines en maintenant un registre « littéraire » (Woodmansee et Jaszi, 1994) : l'écriture et la responsabilité y sont considérées comme absolues. Le travail de définition consiste ici à étendre et à ajuster les registres de l'auteur aux pratiques collectives de recherche et de signature.

En sciences biomédicales, l'accent est davantage mis sur le niveau individuel dans la production collective. C'est le crédit et la responsabilité de chaque participant qui est privilégié. Le crédit est défini de manière large, il ne se réduit pas aux seules tâches cognitives dans le processus de recherche. La notion d'auteur et la responsabilité absolue qu'elle contient sont cependant rejetées. La responsabilité de l'ensemble de la recherche n'est pas attribuée à chaque cosignataire, mais à un garant. Dans cet espace disciplinaire, les prescriptions tendent donc à construire l'auteur contre sa définition absolue en décloisonnant chaque registre : le crédit est spécifié et la responsabilité divisée. À l'interface des sphères académique et industrielle, les pratiques de recherche en sciences biomédicales sont à l'entre-deux de la paternité (la publication académique) et de la propriété (la prise de brevets). Par ailleurs, les connaissances produites dans ce domaine sont fortement liées à des questions d'éthique et de santé publique. De ce fait elles sont fortement soumises à des exigences de traçabilité. Le développement de la description des contributions (contributorship) peut ainsi être compris comme une manière spécifique d'expliciter tant la propriété et la responsabilité de chaque contributeur que la traçabilité du projet de recherche.

Ces modalités particulières d'articulation du crédit et de la responsabilité mettent donc en évidence le rôle des espaces disciplinaires dans la définition de l'auctorialité en science ${ }^{9}$. Ces différences sont significatives : elles montrent que la question de l'auteur n'est pas purement théorique. Elle est aussi une question pratique, qui « ne peut être rapportée ni à une seule détermination ni à un unique moment historique " (Chartier, 1996: 72). Loin d'une simple application de registres qui composent historiquement la notion d'auteur, ou d'une définition a priori, l'activité scientifique requiert en ce domaine des ajustements, voire des innovations. Attribuer le statut de non-auteurs aux scientifiques évacue d'une autre façon la question de leur présence dans leurs productions. Cette présence, activée par la signature, est pourtant bien effective : c'est par elle que s'opère l'attribution du crédit et de la responsabilité. Mais c'est également cette présence de l'auteur, comme personne autorisée ayant une position sociale particulière, qui confère autorité au discours de connaissance (Shapin, 1994 ; Licoppe, 1996).

Prendre au sérieux la question de l'auteur en science consiste donc à interroger les modalités particulières qui conduisent à des formes d'appropriation spécifiques, mais

9. Comparé à l'analyse menée ici, le travail de Galison (1997) sur l'auteur en physique montre combien chaque espace disciplinaire définit de manière spécifique l'opérationnalité de cette notion. 
également multiples, dans chaque espace disciplinaire. Une telle perspective oblige à considérer différents niveaux d'investigation : la nature (plus fondamentale ou plus appliquée) des savoirs produits au sein d'un espace disciplinaire ; l'organisation sociocognitive de la recherche et plus spécifiquement les compétences (mono ou multidisciplinaires) qui y sont requises, tout comme le rôle des éditeurs dans le cadrage des pratiques ; mais également les liens tissés entre univers académique, industriel, et gouvernemental, et la place de cet espace dans cette triade. De ces divers niveaux dépendent les formes d'appropriation possibles, et donc la définition spécifique de l'auteur qui peut en découler. C'est au prix d'un tel travail nourri empiriquement qu'une éventuelle construction théorique plus générale de l'« auteur scientifique » pourra être formulée.

\section{Références}

American Psychological Association. (1992) "Ethical principles of psychologists and code of conduct" American Psychologist 47: 1597-1611.

American Psychological Association. (1994) Publication Manual. Washington, DC: American Psychological Association.

Bazerman, C. (1988) Shaping written knowledge: the genre and activity of the experimental article in science. Madison, Wisconsin: The University of Wisconsin Press.

Bhopal, R., Rankin, J., McColl, E., Thomas, L.H., Kaner, E., Stacy, R., Pearson, P., Vernon, B. et Rodgers, H. (1997) "The vexed question of authorship: views of researchers in British Medical faculty" British Medical Journal 314(7086): 1009-1012.

Biagioli, M. (1999) "Aporias of scientific authorship. Credit and responsibility in contemporary biomedicine" In M. Biagioli (ed.) The science studies reader. New York: Routledge, pp. 12-30.

Bridgwater, C.A., Bornstein, P.H. et Walkenbach, J. (1981) "Ethical issues in the assignment of publication credit" American Psychologist 36: 524-525.

Broad, W. et Wade, N. (1987) La Souris truquée, enquête sur la fraude scientifique. Paris: Seuil.

Chartier, R. (1996) "Figures de l'auteur" In Culture écrite et société. L'ordre des livres (XIVe XVIIle siècle). Paris: Albin Michel, pp. 45-80.

Couturier, M. (1995) La Figure de l'auteur. Paris: Seuil.

Digiusto, E. (1994) "Equity in authorship: a strategy for assigning credit when publishing" Social Science and Medicine 38(1): 55-58.

Drenth, J.P.H. (1998) "Multiple authorship: the contribution of senior authors" Journal of the American Medical Association 280(3): 219-221.

Eastwood, S., Derish, P., Leash, E. et Ordway, S. (1996) "Ethical issues in biomedical research: perceptions and practices of postdoctoral research fellows responding to a survey" Sci Eng Ethics 2: 89-114.

Eisenstein, E.L. (1991) La Révolution de l'imprimé dans l'Europe des premiers temps modernes. Paris: La Découverte.

Endersby, J.W. (1996) "Collaborative research in the social sciences: multiple authorship and publication credit" Social Science Quarterly 77(2): 375-392.

Engler, R.L., Covell, J.W., Friedman, P.J., Kitcher, P.S. et Peters, R.M. (1987)

"Misrepresentation and responsibility in medical research" New England Journal of Medicine 317: 1383-1389. 
Epstein, R.J. (1993) "Six authors in search of a citation: villains or victims of the Vancouver convention?" British Medical Journal 306: 765.

Fine, M.A. et Kurdek, L.A. (1993) "Reflections on determining authorship credit and authorship order on faculty-student collaborations" American Psychologist 48(11): 1141-1147.

Flanagin, A., Carey, L.A. et Fontanarosa, P.B. (1998) "Prevalence of articles with honorary authors and ghost authors in peer-reviewed medical journal" Journal of the American Medical Association 280(3): 222-224.

Foucault, M. (1969) “Qu'est-ce qu'un auteur ?” Bulletin de la Société Française de Philosophie 63(3): 73-104.

Fraenkel, B. (1992) La Signature: genèse d'un signe. Paris: Gallimard, Bibliothèque des Histoires.

Fraenkel, B. (1997) " "Répondre à tous". Une enquête sur le service du courrier présidentiel” In D. Fabre (ed.) Par Ecrit. Ethnologie des écritures quotidiennes. Paris: MSH, pp. 243-271.

Galison, P. (1997) "The collective author" Papier présenté au meeting intitulé 'What is a scientific author?', Harvard University, 7-9 Mars.

Godlee, F. (1996) "Definition of "authorship" may be changed" British Medical Journal 312: 1501-1502.

Goodman, N.W. (1994) "Survey of fulfillment of criteria for authorship in published medical research" British Medical Journal 309: 1482.

Hagstrom, W.O. (1982) "Gift giving as an organizing principle in science" In B. Barnes et D. Edge (eds.) Science in context. The Open University Press, pp. 21-34.

Hoen, W.P., Walvoort, H.C. et Overbeke, A.J. (1998) "What are the factors determining authorship and the order of the authors'names: a study among authors of the Nederlands Tijdschrift voor Geneeskunde (Dutch Journal of Medicine)" Journal of the American Medical Association 280(3): 217-218.

Horton, R. et Smith, R. (1996) "Time to redefine authorship" British Medical Journal 312(7033): 723.

Huth, E.J. (1986) "Abuses and uses of authorship" Annals of Internal Medicine 104: 266-267.

International Committee of Medical Journal Editors. (1985) "Guidelines on authorship" British Medical Journal 291: 722.

International Committee of Medical Journal Editors. (1993) "Uniform requirements for manuscripts submitted to biomedical journals" Journal of the American Medical Association 269: 2282-2286.

International Committee of Medical Journal Editors. (1997) "Uniform requirements for manuscripts submitted to biomedical journals" Journal of the American Medical Association 277: 927-934.

International Committee of Medical Journal Editors. (2000) "Uniform requirements for manuscripts submitted to biomedical journals" (http://www.acponline.org/journals/resource/ unifreqr.htm).

Leash, E. (1997) "Is it time for a new approach to authorship?" Journal of Dental Research 76(3): 724-727.

Licoppe, C. (1996) La Formation de la pratique scientifique. Le discours de l'expérience en France et en Angleterre (1630-1820). Paris: La Découverte.

Luukkonen, T., Persson, O. et Sivertsen, G. (1992) "Understanding patterns of international scientific collaboration" Science, Technology \& Human Values, 17(1): 101-126.

Merton, R.K. (1968) "The Matthew effect in science" Science 159(3810): 56-63. 
Moulopoulos, S.D., Sideras, D.A. et Georgilis, K.A. (1983) "Individual contributions to multiauthor papers" British Medical Journal 287: 1608-10.

Over, R. et Smallman, S. (1973) "Maintenance of individual visibility in publication of collaborative research by psychologists" American Psychologist 28: 161-166.

Pontille, D. (2000) La Signature scientifique: espaces d'inscription et mises en ordre. Thèse nouveau régime, département de sociologie. Toulouse: Université Toulouse II.

Rennie, D. (1999) "Solutions" In M. Biagioli, J. Crane, P. Derish, M. Gruber, D. Rennie et R. Horton (eds.) CBE task force on authorship: draft white paper. (http://www.cbe.org/ATF.html)

Rennie, D. et Flanagin, A. (1994) "Authorship! Authorship! Ghosts, guests, and grafters, and the two-sided coin" Journal of the American Medical Association 271: 469-471.

Rennie, D., Yank, V. et Emanuel, L. (1997) "When authorship fails: a proposal to make contributors accountable" Journal of the American Medical Association 278: 579-585.

Saffran, M. (1989) "On multiple authorship: describe the contribution" The Scientist 2: 9.

Shapin, S. (1989) "The invisible technician” American Scientist 77: 554-563.

Shapin, S. (1994) A social history of truth: civility and science in seventeenth-century. Chicago: The University of Chicago Press.

Shapiro, D.W., Wenger, N.S. et Shapiro, M.F. (1994) "The contributions of authors to multiauthored biomedical research papers" Journal of the American Medical Association 271: 483-442.

Smith, J. (1994) "Gift authorship: a poisoned chalice?” British Medical Journal 309: 1456-57.

Smith, R. (1997a) "Authorship is dying: long live contributorship" British Medical Journal 315(7110): Editorial.

Smith, R. (1997b) "Authorship: time for a paradigm shift?" British Medical Journal 314(7086): 992.

Spiegel, D. et Keith-Spiegel, P. (1970) "Assignment of publication credits: ethics and practices of psychologists" American Psychologist 25: 738-747.

Stephan, P.E. et Levin, S.G. (1997) "The critical importance of careers in collaborative scientific research" Revue d'Economie Industrielle 79(1): 45-61.

Woodmansee, M. et Jaszi, P. (eds.) (1994) The construction of authorship. Textual appropriation in law and literature. Durham and London: Duke University Press.

Yank, V. et Rennie, D. (1999) "Disclosure of researcher contributions: a study of original research articles in The Lancet" Annals of Internal Medicine 130: 661-670.

Zuckerman, H.A. (1968) "Patterns of name ordering among authors of scientific papers: a study of social symbolism and its ambiguity" American Journal of Sociology 74(3): 276-291. Zuckerman, H.A. et Merton, R.K. (1971) "Patterns of evaluation in science: institutionalization, structure and functions of the referee system" Minerva 9(1): 66-100. 